\title{
Are Measures of Left Ventricular Longitudinal Shortening Affected by Left Atrial Enlargement?
}

\author{
Angel Lopez-Candales ${ }^{\mathrm{a}, \mathrm{e}}$, Dagmar F. Hernandez-Suarez ${ }^{\mathrm{b}}$, \\ Francisco Lopez Menendez ${ }^{\mathrm{c}}$
}

\begin{abstract}
Background: Even though left atrial (LA) size and function are intimately related to left ventricular (LV) diastolic dysfunction, the role of LA with regard to LV systolic function is less clear. Consequently, we examined the potential association that might exist between measures of longitudinal LV systolic shortening and LA dilation using LA volume index (LAVI).
\end{abstract}

Methods: In this retrospective analysis, data from 75 echocardiograms (mean age $53 \pm 14$; range 24 - 89 years; mean body surface area (BSA) $2.0 \pm 0.3$ ) were analyzed.

Results: Peak global longitudinal (PGLS) correlated best with LV mass index (LVMI) followed by mitral annular systolic excursion (MAPSE), and age. Similar results were obtained when analyzing the best variables that correlated with LAVI. Finally, MAPSE correlated best with PGLS, then with MA tissue Doppler systolic velocity, BSA, and LAVI in that order. All patients had normal LV ejection fraction (LVEF) and normal sinus rhythm when studied.

Conclusions: LAVI does not directly affect LV systolic function and longitudinal measures of LV shortening are mainly dependent on LV mass. Additional studies are now required to determine how these associations vary when different degrees of LV dilatation and systolic dysfunction are included in the analysis.

Keywords: Automated functional imaging; Left ventricular function; Left atrial enlargement; Left ventricular mass; Left ventricular hypertrophy; Strain imaging

Manuscript submitted November 20, 2017, accepted November 30, 2017

${ }^{a}$ Cardiovascular Medicine Division, School of Medicine, University of Puerto Rico, San Juan, Puerto Rico

${ }^{b}$ School of Medicine, University of Puerto Rico, San Juan, Puerto Rico 'Division of Cardiovascular Health and Disease, University of Cincinnati College of Medicine, Cincinnati, OH, USA

${ }^{\mathrm{e}}$ Corresponding Author: Angel Lopez-Candales, Cardiovascular Medicine Division, School of Medicine, University of Puerto Rico, Medical Sciences Building, PO Box 365067, San Juan, 00936-5067, Puerto Rico.

Email: angel.lopez17@upr.edu

doi: https://doi.org/10.14740/cr637w

\section{Introduction}

The left atrium (LA) not only modulates left ventricular (LV) filling but also cardiovascular performance [1]. Emerging data have clearly depicted the overall contribution of LA performance in predicting adverse cardiovascular events in patients with atrial fibrillation, cardiomyopathy, ischemic heart disease, valvulopathy as well as the general population [2]. Of particular relevance to our study is the considerable evidence supporting the role that an increased LA volume index (LAVI) has in predicting the development of new heart failure irrespective of left ventricular (LV) systolic function $[3,4]$.

Furthermore, not only LA dimension is a recognized predictor of mortality and heart failure hospitalization after adjusting for LV ejection fraction (LVEF) and New York Heart Association functional class in the SOLVD (Studies of Left Ventricular Dysfunction) trials [5], but also LA area was a powerful predictor of death or hospitalization for heart failure independent of age, New York Heart Association functional class, LVEF, and restrictive filling pattern in the MeRGE (Meta-Analysis Research Group in Echocardiography) metaanalysis [6].

However, the LA cannot be in isolation as it shares the mitral annulus (MA) with the LV [7, 8]. This atrio-ventricular interdependence though more closely coupled on the right than on the left side, is likely dependent on longitudinal basal to apical motion [8]. With regard to LV longitudinal shortening, both mitral annular plane systolic excursion (MAPSE) and peak global longitudinal strain (PGLS) are well-validated surrogate measures of LV systolic function [9-13]. In terms of the LV, the association between MAPSE and LVEF is valid only in patients with either normal or dilated $\operatorname{LV}[14,15]$ as MAPSE loses its value when assessing LVEF in patients with LVH since longitudinal shortening is compromised in these patients [16]. Finally, longitudinal shortening is reduced as result of LV hypertrophy (LVH) [17-21], despite normal LVEF [20-24].

However, none of these measures of longitudinal shortening have been examined with regard to the LA. Consequently, we decided to assess the potential impact that LAVI might have on both MAPSE and PGLS measured by automated functional imaging (AFI). To eliminate additional confounders, only patients with normal LVEF and in normal sinus rhythm were 
Table 1. Most Common Clinical Entities for Which the Study Population Had an Echocardiogram

\begin{tabular}{ll}
\hline Clinical condition & Number of patients \\
\hline Hypertension & 47 \\
Diabetes mellitus & 23 \\
Chronic kidney disease/end-stage renal failure & 16 \\
Coronary artery disease & 13 \\
Acute respiratory issues & 13 \\
Other conditions (rheumatic/hematologic/gastrointestinal) & 12 \\
Transient ischemic attack/cerebrovascular accident & 8 \\
Chest pain & 8 \\
Heart failure & 8 \\
Pre operative exam & 6 \\
Cancer & 5 \\
Obstructive sleep apnea & 5 \\
Chronic obstructive pulmonary disease & 4 \\
Syncope & 3 \\
Valvular heart disease & 2 \\
\hline
\end{tabular}

studied.

\section{Methods}

For this retrospective study, our University Hospital Medical Center, Cincinnati, $\mathrm{OH}$ echocardiography database was queried for complete studies that included complete LV AFI acquisition, good endocardial LA and LV border resolution from the parasternal long axis, two- and four-chamber apical views. In addition to the already mentioned study inclusion criteria, studies were excluded if any wall motion abnormalities were present; patients had history of previous cardiac surgery; presence of a wide QRS or conduction block; more than mild valvular regurgitation or stenosis; any ectopy; arrhythmias or heart rate greater than 100 beats per minute. The University of Cincinnati Institutional Review Board (IRB \#12061302) approved the study.

Two-dimensional echocardiographic studies were performed using commercially available systems (Vivid 7 and 9, GE Medical Systems, Milwaukee, WI, USA). Images were obtained in left lateral decubitus position with the patient in the supine position using a $3.5-\mathrm{MHz}$ transducer. Standard two-dimensional, color, pulsed, and continuous-wave Doppler data were digitally acquired in gently held end-expiration, and saved in regular cine loop format for subsequent offline analysis (EchoPAC version 111.0.00; GE-Vingmed Ultrasound AS).

For the purpose of this study, LV end-systolic and end-diastolic volumes were measured as recommended by the American Society of Echocardiography to determine LVEF using the Simpson's method [25]. In addition, surrogate measures of LV systolic function such as MAPSE as well as its systolic velocity of the lateral portion of the mitral annulus were acquired as previously described $[7,8,12,25,26]$. LA volumes were calculated using the biplane area-length formula while
LV mass was determined using M-mode echocardiography, as previously described [25]. Both LA volumes and LV mass were corrected for body surface area (BSA) [25].

Finally, peak systolic strain was measured using GE (General Electric, Medical Systems, Milwaukee, WI, USA) AFI software to analyze speckle-tracking motion of ventricular segments. Specifically, the three-click method has been found useful in minimizing variability as previously reported [23, $27,28]$. In doing so, endocardial borders are automatically tracked throughout the cardiac cycle once a reliable endocardial tracing over 1 frame has been manually drawn. Ultrasound speckles in the image are tracked and two-dimensional strain is obtained by comparing the displacement of the speckles in relation to one another along the endocardial contour throughout the cardiac cycle. The reported PGLS represents the average of individual peak strain measurements from the parasternal long axis, two- and four-apical chamber views.

All continuous data are presented as mean $\pm \mathrm{SD}$. Categorical data are presented as frequencies or percentages. Correlations between measurements were performed using Pearson's correlation. Multivariate logistic regression analysis was performed to detect the best identifying measured variables. Pvalues of $<0.05$ were considered to be statistically significant. All analyses were performed using SPSS version 17.0 for Windows (SPSS, Inc., Chicago, IL).

\section{Results}

The studied population included data from 75 patients (mean age $53 \pm 14$; range $24-89$ years, with a mean BSA $2.0 \pm 0.3$ ).

The most common clinical entities for which our patients were studied with echocardiography are depicted in Table 1.

In terms of the studied echocardiographic variables, the following are mean and range values for LAVI $28 \pm 12 \mathrm{~mL} / \mathrm{m}^{2}$ 
Table 2. Stepwise Multiple Regression Analysis to Assess Which Variable Was Best to Identify MAPSE

\begin{tabular}{lllll}
\hline Independent variables & Coefficient & Std. error & $\mathbf{r}$ & P \\
\hline Age & -0.002601 & 0.001640 & -0.3480 & 0.1175 \\
BSA & 0.1929 & 0.06801 & 0.1720 & 0.0013 \\
LAVI & 0.005699 & 0.001840 & 0.08733 & 0.0026 \\
LVMI & 0.0001815 & 0.0008585 & -0.3783 & 0.8332 \\
MA TDI S' & 0.03845 & 0.009917 & 0.4686 & 0.0001 \\
PGLS & -0.02467 & 0.007077 & -0.5419 & $<0.0001$ \\
\hline
\end{tabular}

BSA: body surface area; LAVI: left atrial volume index; LVMI: left ventricular mass index; MAPSE: mitral annular plane systolic excursion; MA TDI S': mitral annular tissue Doppler imaging systolic velocity'; PGLS: peak global longitudinal strain.

Table 3. Stepwise Multiple Regression Analysis to Assess Which Variable Was Best to Identify PGLS

\begin{tabular}{lllll}
\hline Independent variables & Coefficient & Std. error & $\mathbf{r}$ & $\mathbf{P}$ \\
\hline Age & 0.05185 & 0.02560 & 0.3263 & 0.0486 \\
BSA & 1.6768 & 1.1167 & 0.02060 & 0.1378 \\
LAVI & 0.01874 & 0.03094 & 0.2141 & 0.5467 \\
LVMI & 0.06193 & 0.01128 & 0.6623 & $<0.0001$ \\
MAPSE & -6.1455 & 1.7628 & -0.5419 & 0.0026 \\
MA TDI S' & 0.2365 & 0.1706 & -0.3974 & 0.1700 \\
\hline
\end{tabular}

(range: 11 - 87); LV mass index (LVMI) $99 \pm 37 \mathrm{~g} / \mathrm{m}^{2}$ (range: 42 - 218); MAPSE $1.3 \pm 0.3 \mathrm{~cm}$ (range: 0.7 - 1.9); mitral annular tissue Doppler imaging systolic velocity (MA TDI S') $8.3 \pm 2.6$ (range: $4-14$ ) and PGLS $-16 \pm 4$ (range: -9 to -25 ).

We first conducted a stepwise multiple regression analysis to determine which of the collected variables from this study population correlated with MAPSE and found that PGLS was the best independent variable followed by MA TDI S', BSA and LAVI in that order as seen in Table 2.

We then conducted a different stepwise multiple regression and this time examined which of these variables correlated best with PGLS. In this analysis, we found that LVMI was the best independent variable followed by MAPSE, and age in that order as seen in Table 3.

Finally, we conducted a different stepwise multiple regression and this time examined which of these variables correlated best with LAVI. In this analysis we found that LVMI was the best independent variable followed by MAPSE, and age in that order as seen in Table 4.

Intraobserver as well as interobserver PGLS reproducibility for our laboratory measurements have been previously reported [24].

\section{Discussion}

Though LA size and function have been recognized as critical components in mediating LV diastolic function; the potential role of the LA in terms of LV systolic function is less clear. The findings of our study seem to suggest that LAVI does not affect LV systolic function given the lack of a correlation between LAVI and PGLS and MA TDI S'. However, we still need to explain the observed results with regard to LAVI and MAPSE.

It is well established anatomically speaking the LV is comprised of three continuous layers of muscle bundles [29-31]. Furthermore, helical arrangement of these muscle fibers that are anchored at a relatively stationary apex is required for effective systolic ejection of the LV due to contribution of longitudinal as well as circumferential shortening of these fibers [32-34]. Even though the LA has not been included in this mechanical paradigm, not only the continuity of this chamber to the $\mathrm{LV}$, but also sharing the MA makes the anatomical location

Table 4. Stepwise Multiple Regression Analysis to Assess Which Variable Was Best to Identify LAVI

\begin{tabular}{lllll}
\hline Independent variables & Coefficient & Std. error & $\mathbf{r}$ & P \\
\hline Age & 0.1770 & 0.1008 & 0.1802 & 0.0282 \\
BSA & -5.3373 & 4.3896 & -0.05827 & 0.2282 \\
LVMI & 0.1111 & 0.05124 & 0.3448 & 0.0002 \\
MAPSE & 21.6913 & 7.0041 & 0.08733 & 0.0054 \\
MA TDI S, & -0.7345 & 0.6702 & -0.1761 & 0.2769 \\
PGLS & 0.2864 & 0.4728 & 0.2141 & 0.5467 \\
\hline
\end{tabular}


of the LA critical to examine its potential role in LV systolic function.

From a mechanistic point of view, it makes perfect sense to examine the potential role of the LA with regard to the LV based on the conceptual approximation of the heart as a cylinder when examining longitudinal measures of LV systolic function such as GPLS, MAPSE and MA TDI S' since this in patients with an increased LV mass index [35]. In this physiologic construct, the external LV radius is fixed with a time-varying internal radius and height as the MA moves from base to apex and vice versa [35]; therefore, in this model, the LA should contribute in some fashion to this longitudinal LV shortening. However, our results disprove this association. Even though there is a close interrelationship between LA and LV to maintain optimal cardiac performance; the LA appears simply to modulate LV filling through its reservoir, conduit, and booster pump function [36]. Furthermore, LV function also influences LA function throughout the cardiac cycle [36]. An increased LVMI will certainly increase LV filling pressure and consequently worsen LV diastolic function. At this point, the limits of LA preload reserve are reached and the LA would simply function as a conduit [36]. Therefore, the overall impact of LAVI on left atrioventricular plane displacement or LV shortening should be negligible as the main contributor of this LV basal to apex motion is myocardial fiber contractility that appears to be only mediated by LV geometry and LV hypertrophy [14-16]. Consequently, as seen by our results, there should not be any correlation between LAVI and longitudinal measures of LV shortening. Though LAVI did not correlate with MA TDI S' and PGLS, it correlated with MAPSE. We speculate that this association (Table 4) is strictly based on anatomical basis between LA and LV through the MA. As expected, overall extent of MA motion would be ultimately dictated by predominant LB muscle fibers, degree of LV mass that will limit magnitude of shortening and pressure differences between both chambers rather than LA size.

However, the following limitations need to be acknowledged. First, the retrospective nature of the study. Second, the small size and heterogeneity of our sample. However, the main goal of this study was to assess the potential impact that LAVI might have on both MAPSE and PGLS. Second, patients with cardiac rhythms besides normal sinus rhythm were not included. However, the presence of other rhythms besides normal sinus rhythm would have affected interpretation of LV longitudinal shortening and interfere with assessment of LAVI. Third, our patient population had no invasive hemodynamic data; hence, some potentially valuable information that could have improved our correlations was not included. However, the purpose was to assess impact of LAVI on LV shortening measured by echocardiography. Fourth, speckle tracking imaging data were not included. Certainly all three LA function parameters would have been useful when analyzing LV shortening. In fact, the potential role of LA dysfunction in the pathophysiology of LV diastolic dysfunction is an old concept [37]. In this scenario, normal subjects and asymptomatic hypertensive patients both are able to increase their late diastolic MA velocities during exercise while patients with heart failure and preserved ejection fraction are unable to benefit from this mechanism [37]. Finally, only patients with a normal LVEF based on Simpson's were included. Therefore, we are unable to make any comments regarding any of the observed associations with different degrees of LV systolic dysfunction.

\section{Conclusion}

Even though LA size and function have been recognized as important variables mediating LV diastolic function, our results tend to suggest that LAVI does not directly affect LV systolic function and longitudinal measures of LV shortening are mainly mediated by LV mass. Additional studies are now required to determine how different degrees of LV dilatation and systolic dysfunction affect the observed associations shown in this analysis.

\section{Acknowledgments}

This publication was partially supported by the National Institute of Health Award Numbers R25MD007607, S21MD001830 and TL1 TR001434-3. Its contents are solely the responsibility of the authors and do not necessarily represent the official views of the National Institutes of Health.

\section{Conflict of Interest}

None.

\section{References}

1. Barbier P, Solomon SB, Schiller NB, Glantz SA. Left atrial relaxation and left ventricular systolic function determine left atrial reservoir function. Circulation. 1999;100(4):427-436.

2. Hoit BD. Left atrial size and function: role in prognosis. $\mathrm{J}$ Am Coll Cardiol. 2014;63(6):493-505.

3. Takemoto Y, Barnes ME, Seward JB, Lester SJ, Appleton CA, Gersh BJ, Bailey KR, et al. Usefulness of left atrial volume in predicting first congestive heart failure in patients $>$ or $=65$ years of age with well-preserved left ventricular systolic function. Am J Cardiol. 2005;96(6):832836.

4. Gottdiener JS, Kitzman DW, Aurigemma GP, Arnold AM, Manolio TA. Left atrial volume, geometry, and function in systolic and diastolic heart failure of persons $>$ or $=65$ years of age (the cardiovascular health study). Am J Cardiol. 2006;97(1):83-89.

5. Quinones MA, Greenberg BH, Kopelen HA, Koilpillai C, Limacher MC, Shindler DM, Shelton BJ, et al. Echocardiographic predictors of clinical outcome in patients with left ventricular dysfunction enrolled in the SOLVD registry and trials: significance of left ventricular hypertrophy. Studies of Left Ventricular Dysfunction. J Am Coll Cardiol. 2000;35(5):1237-1244.

6. Rossi A, Temporelli PL, Quintana M, Dini FL, Ghio S, 
Hillis GS, Klein AL, et al. Independent relationship of left atrial size and mortality in patients with heart failure: an individual patient meta-analysis of longitudinal data (MeRGE Heart Failure). Eur J Heart Fail. 2009;11(10):929-936.

7. Lopez-Candales A, Rajagopalan N, Gulyasy B, Edelman $\mathrm{K}$, Bazaz R. Comparative echocardiographic analysis of mitral and tricuspid annular motion: differences explained with proposed anatomic-structural correlates. Echocardiography. 2007;24(4):353-359.

8. Bazaz R, Edelman K, Gulyasy B, Lopez-Candales A. Evidence of robust coupling of atrioventricular mechanical function of the right side of the heart: insights from M-mode analysis of annular motion. Echocardiography. 2008;25(6):557-561.

9. Carlsson M, Ugander M, Mosen H, Buhre T, Arheden H. Atrioventricular plane displacement is the major contributor to left ventricular pumping in healthy adults, athletes, and patients with dilated cardiomyopathy. Am J Physiol Heart Circ Physiol. 2007;292(3):H1452-1459.

10. Jones CJ, Raposo L, Gibson DG. Functional importance of the long axis dynamics of the human left ventricle. $\mathrm{Br}$ Heart J. 1990;63(4):215-220.

11. Simonson JS, Schiller NB. Descent of the base of the left ventricle: an echocardiographic index of left ventricular function. J Am Soc Echocardiogr. 1989;2(1):25-35.

12. Matos J, Kronzon I, Panagopoulos G, Perk G. Mitral annular plane systolic excursion as a surrogate for left ventricular ejection fraction. J Am Soc Echocardiogr. 2012;25(9):969-974.

13. Geyer H, Caracciolo G, Abe H, Wilansky S, Carerj S, Gentile F, Nesser HJ, et al. Assessment of myocardial mechanics using speckle tracking echocardiography: fundamentals and clinical applications. J Am Soc Echocardiogr. 2010;23(4):351-369; quiz 453-355.

14. Hoffman EA, Ritman EL. Invariant total heart volume in the intact thorax. Am J Physiol. 1985;249(4 Pt 2):H883890.

15. Carlhall CJ, Lindstrom L, Wranne B, Nylander E. Atrioventricular plane displacement correlates closely to circulatory dimensions but not to ejection fraction in normal young subjects. Clin Physiol. 2001;21(5):621-628.

16. Wandt B, Bojo L, Tolagen K, Wranne B. Echocardiographic assessment of ejection fraction in left ventricular hypertrophy. Heart. 1999;82(2):192-198.

17. Onose Y, Oki T, Mishiro Y, Yamada H, Abe M, Manabe $\mathrm{K}$, Kageji $\mathrm{Y}$, et al. Influence of aging on systolic left ventricular wall motion velocities along the long and short axes in clinically normal patients determined by pulsed tissue doppler imaging. J Am Soc Echocardiogr. 1999;12(11):921-926.

18. Mishiro Y, Oki T, Yamada H, Onose Y, Matsuoka M, Tabata T, Wakatsuki T, et al. Use of angiotensin II stress pulsed tissue Doppler imaging to evaluate regional left ventricular contractility in patients with hypertrophic cardiomyopathy. J Am Soc Echocardiogr. 2000;13(12):10651073.

19. Koulouris SN, Kostopoulos KG, Triantafyllou KA, Karabinos I, Bouki TP, Karvounis HI, Omran H, et al. Im- paired systolic dysfunction of left ventricular longitudinal fibers: a sign of early hypertensive cardiomyopathy. Clin Cardiol. 2005;28(6):282-286.

20. Fang ZY, Leano R, Marwick TH. Relationship between longitudinal and radial contractility in subclinical diabetic heart disease. Clin Sci (Lond). 2004;106(1):53-60.

21. Yu CM, Lin H, Yang H, Kong SL, Zhang Q, Lee SW. Progression of systolic abnormalities in patients with "isolated" diastolic heart failure and diastolic dysfunction. Circulation. 2002;105(10):1195-1201.

22. Palmon LC, Reichek N, Yeon SB, Clark NR, Brownson D, Hoffman E, Axel L. Intramural myocardial shortening in hypertensive left ventricular hypertrophy with normal pump function. Circulation. 1994;89(1):122-131.

23. Lopez-Candales A. Automated functional imaging for assessment of left ventricular mechanics in the presence of left ventricular hypertrophy. Echocardiography. 2014;31(5):605-614.

24. Maclver DH, Adeniran I, Zhang H. Left ventricular ejection fraction is determined by both global myocardial strain and wall thickness. Int $\mathrm{J}$ of Cardiology Heart \& Vasculature. 2015;7:113-118.

25. Lang RM, Badano LP, Mor-Avi V, Afilalo J, Armstrong A, Ernande L, Flachskampf FA, et al. Recommendations for cardiac chamber quantification by echocardiography in adults: an update from the American Society of Echocardiography and the European Association of Cardiovascular Imaging. J Am Soc Echocardiogr. 2015;28(1):139 e14.

26. Saxena N, Rajagopalan N, Edelman K, Lopez-Candales A. Tricuspid annular systolic velocity: a useful measurement in determining right ventricular systolic function regardless of pulmonary artery pressures. Echocardiography. 2006;23(9):750-755

27. Feigenbaum H, Mastouri R, Sawada S. A practical approach to using strain echocardiography to evaluate the left ventricle. Circ J. 2012;76(7):1550-1555.

28. Delgado V, Mollema SA, Ypenburg C, Tops LF, van der Wall EE, Schalij MJ, Bax JJ. Relation between global left ventricular longitudinal strain assessed with novel automated function imaging and biplane left ventricular ejection fraction in patients with coronary artery disease. J Am Soc Echocardiogr. 2008;21(11):1244-1250.

29. MacIver DH. A mathematical model of left ventricular contraction and its application in heart disease. In: Atherton M, Collins M, Dayer M, editors. Repair and redesign of physiological systems. Boston: WIT Press; 2008. pp. 65-86.

30. MacIver DH. Current controversies in heart failure with a preserved ejection fraction. Future Cardiol. 2010;6(1):97111.

31. Anderson RH, Ho SY, Redmann K, Sanchez-Quintana $\mathrm{D}$, Lunkenheimer PP. The anatomical arrangement of the myocardial cells making up the ventricular mass. Eur J Cardiothorac Surg. 2005;28(4):517-525.

32. Greenbaum RA, Ho SY, Gibson DG, Becker AE, Anderson RH. Left ventricular fibre architecture in man. $\mathrm{Br}$ Heart J. 1981;45(3):248-263.

33. Torrent-Guasp F, Kocica MJ, Corno AF, Komeda M, Car- 
reras-Costa F, Flotats A, Cosin-Aguillar J, et al. Towards new understanding of the heart structure and function. Eur J Cardiothorac Surg. 2005;27(2):191-201.

34. MacIver DH. Understanding myocardial deformation, 'global systolic function' and abnormal geometry. Heart. 2009.

35. Ghosh E, Shmuylovich L, Kovacs SJ. Vortex formation time-to-left ventricular early rapid filling relation: model-based prediction with echocardiographic validation. J
Appl Physiol (1985). 2010;109(6):1812-1819.

36. Rosca M, Lancellotti P, Popescu BA, Pierard LA. Left atrial function: pathophysiology, echocardiographic assessment, and clinical applications. Heart. 2011;97(23):19821989.

37. Emilsson K, Brudin L, Wandt B. The mode of left ventricular pumping: is there an outer contour change in addition to the atrioventricular plane displacement? Clin Physiol. 2001;21(4):437-446. 